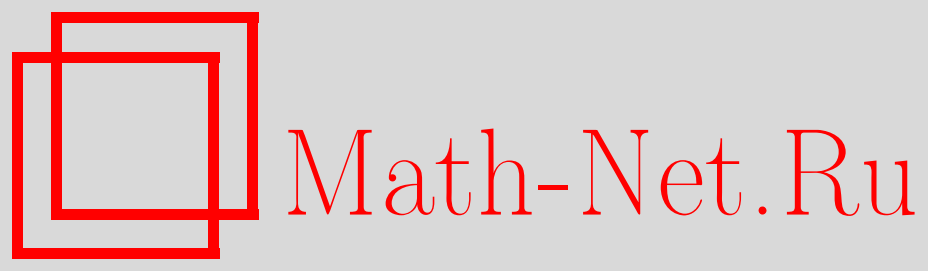

Д. Л. Хебгуд, И. Г. Тодоров, О непрерывности носителя бимодулей над максимальными коммутативными самосопряженными алгебрами, Функи. анализ и его прил., 2008, том 42, выпуск 3, 93-95

DOI: https://doi.org/10.4213/faa2920

Использование Общероссийского математического портала MathNet.Ru подразумевает, что вы прочитали и согласны с пользовательским соглашением

http: //www.mathnet.ru/rus/agreement

Параметры загрузки:

IP : 3.95 .254 .165

26 апреля 2023 г., $17: 52: 21$

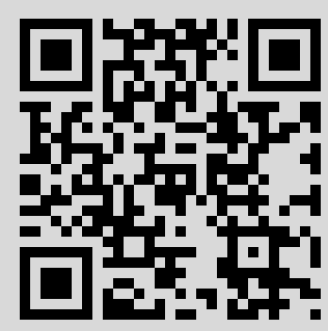




\title{
О непрерывности носителя бимодулей над максимальными коммутативными самосопряженными алгебрами
}

\author{
(c) 2008. Дж. Л. ХЕБгуд, И. Г. Тодоров
}

Для заданной алгебры (или множества) операторов $\mathscr{A} \subseteq \mathscr{B}(\mathscr{H})$ обозначим через Lat $\mathscr{A}$ множество всех замкнутых инвариантных подпространств в $\mathscr{A}$. Вопрос о непрерывной зависимости множества Lat $\mathscr{A}$ от $\mathscr{A}$ изучали Дэвидсон, Халмош, Шульман и другие авторы. В данной работе рассматривается аналогичный вопрос для подпространств операторов, представляющих собой бимодули над двумя максимальными коммутативными самосопряженными алгебрами (м.к.c.a.). Наш подход опирается на понятия бирешетки и носителя таких объектов, введенные в [2] и [7]. Доказательства анонсируемых здесь результатов будут опубликованы в другой работе.

Прежде всего, введем обозначения и напомним некоторые необходимые нам результаты. Пусть $\mathscr{H}$ и $\mathscr{K}$ - гильбертовы пространства. Обозначим через $\mathscr{B}(\mathscr{H}, \mathscr{K})$ пространство всех ограниченных линейных операторов, действующих из $\mathscr{H}$ в $\mathscr{K}$. Пусть $\mathscr{D}_{1}$ и $\mathscr{D}_{2}-$ м.к.с.а. в $\mathscr{H}$ и $\mathscr{K}$ соответственно. $\mathscr{D}_{2}, \mathscr{D}_{1}$-бимодулем (или просто м.к.с.а.-бимодулем, если из контекста понятно, о каких $\mathscr{D}_{1}$ и $\mathscr{D}_{2}$ идет речь) называется такое подпространство $\mathscr{U} \subseteq \mathscr{B}(\mathscr{H}, \mathscr{K})$, что $\mathscr{D}_{2} \mathscr{U} \mathscr{D}_{1} \subseteq \mathscr{U}$. Если $\mathscr{U} \subseteq \mathscr{B}(\mathscr{H}, \mathscr{K})$ - м.к.с.а.-бимодуль, то мы обозначим через $\operatorname{Ball}(\mathscr{U})$ единичный шар в $\mathscr{U}$ и положим

$$
\operatorname{Bil} \mathscr{U}=\left\{(P, Q) \in \mathscr{D}_{1} \times \mathscr{D}_{2}: P \text { и } Q \text { проекторы и } Q \mathscr{U} P=\{0\}\right\} .
$$

Множество $S=\operatorname{Bil} \mathscr{U}$ представляет собой бирешетку [7] в том смысле, что $(P, 0),(0, Q) \in \operatorname{bil} \mathscr{U}$ для любых проекторов $P \in \mathscr{D}_{1}$ и $Q \in \mathscr{D}_{2}$ и что из включений $\left(P_{1}, Q_{1}\right),\left(P_{2}, Q_{2}\right) \in \operatorname{Bil} \mathscr{U}$ вытекает, что $\left(P_{1} \wedge P_{2}, Q_{1} \vee Q_{2}\right),\left(P_{1} \vee P_{2}, Q_{1} \wedge Q_{2}\right) \in$ Bil $\mathscr{U}$. Через Conv $S$ обозначим слабо замкнутую выпуклую оболочку множества $S$.

Пусть $(X, m)$ и $(Y, n)$ - обычные пространства с (конечной) мерой. Положим $\mathscr{H}=L^{2}(X, m)$ и $\mathscr{K}=L^{2}(Y, n)$. Пусть $\mathscr{D}_{1} \equiv L^{\infty}(X, m)$ и $\mathscr{D}_{2} \equiv L^{\infty}(Y, n)-$ м.к.с.а. мультипликаторов в $\mathscr{H}$ и $\mathscr{K}$ соответственно. Пусть $\kappa \subseteq X \times Y-$ измеримое подмножество. Говорят, что носитель оператора $T$ содержится в $\kappa$, если $P(\beta) T P(\alpha)=0$ для любых измеримых подмножеств $\alpha \subseteq X$ и $\beta \subseteq Y$, таких, что $(\alpha \times \beta) \cap \kappa=\varnothing$. (Здесь $P(\gamma)$ - оператор умножения на характеристическую функцию измеримого множества $\gamma$.) Пространство $\mathscr{M}_{\max }(\kappa)$ всех операторов с носителем в $\kappa$ представляет собой рефлексивный м.к.с.а.-бимодуль в смысле [4]. Обратно, в [2] было показано, что любой рефлексивный м.к.с.а.-бимодуль $\mathscr{U}$ может быть представлен в таком виде для некоторого по существу единственного множества $\kappa \subseteq X \times Y$, представляющего собой дополнение счетного объединения измеримых прямоугольников (такие множества будем называть $\omega$-замкнутыми [2]). Множество $\kappa$ называется носителем бимодуля $\mathscr{U}$. Согласно [1] и [7], существует минимальный *-слабо замкнутый м.к.с.а.-бимодуль $\mathscr{U}$, peфлексивная оболочка которого в смысле [4] совпадает с $\mathscr{M}_{\max }(\kappa)$; этот м.к.с.а.бимодуль мы обозначим через $\mathscr{M}_{\min }(\kappa)$. Тогда $\operatorname{Bil} \mathscr{M}_{\max }(\kappa)=\operatorname{Bil} \mathscr{M}_{\min }(\kappa)=S_{\kappa}$, 
где

$$
S_{\kappa}=\{(P(\alpha), P(\beta)): \alpha \subseteq X \text { и } \beta \subseteq Y \text { измеримы и }(\alpha \times \beta) \cap \kappa=\varnothing\} .
$$

Если $\mathscr{M}_{\min }(\kappa)=\mathscr{M}_{\max }(\kappa)$, то будем говорить, что на $\kappa$ выполняется операторный синтез [1].

Пусть $\kappa \subseteq X \times Y$. Хейдон и Шульман [3] определили величину

$$
\gamma(\kappa)=\inf \{m(\alpha)+n(\beta): \kappa \subseteq(\alpha \times Y) \cup(X \times \beta)\},
$$

где точная нижняя грань берется по измеримым множествам $\alpha$ и $\beta$. Они показали, что отображение $\kappa \rightarrow \gamma(\kappa)$ является емкостью на множестве всех подмножеств множества $X \times Y$ в том смысле, что

(a) $\gamma(\kappa) \leqslant \gamma\left(\kappa^{\prime}\right)$, если $\kappa \subseteq \kappa^{\prime}$;

(b) $\lim _{n \rightarrow \infty} \gamma\left(\kappa_{n}\right)=\gamma(\kappa)$, если $\left\{\kappa_{n}\right\}$ - возрастающая последовательность подмножеств в $X \times Y$ и $\kappa=\bigcup \kappa_{n}$;

(c) $\gamma(\kappa)=\inf \{\gamma(U): U$ открыто и $\kappa \subseteq U\}$.

Соотношение (c) выполняется для любой пары топологий на $X$ и $Y$, относительно которых $m$ и $n$ являются регулярными борелевскими мерами.

Пусть $\mathscr{Z}$ - семейство упорядоченных троек вида $\left(\left(\alpha_{i}\right)_{i=1}^{N},\left(\beta_{i}\right)_{i=1}^{N},\left(\mu_{i}\right)_{i=1}^{N}\right)$, где $\left(\alpha_{i}\right)_{i=1}^{N}$ (соответственно $\left.\left(\beta_{i}\right)_{i=1}^{N}\right)-($ упорядоченное) измеримое разбиение множества $X$ (соответственно $Y$ ) и $\mu_{i} \geqslant 0, i=1, \ldots, N$.

Зафиксируем подмножество $\kappa \subseteq X \times Y$. Для любого элемента $\Delta=\left(\left(\alpha_{i}\right)_{i=1}^{N}\right.$, $\left.\left(\beta_{i}\right)_{i=1}^{N},\left(\mu_{i}\right)_{i=1}^{N}\right) \in \mathscr{Z}$ положим

$$
\gamma_{\Delta}(\kappa)=\inf \left\{\sum_{i=1}^{N} \mu_{i}\left(m\left(\alpha \cap \alpha_{i}\right)+n\left(\beta \cap \beta_{i}\right)\right): \kappa \subseteq(\alpha \times Y) \cup(X \times \beta)\right\},
$$

где точная нижняя грань берется по измеримым множествам $\alpha$ и $\beta$. Ясно, что если $\mu_{i}=1$ для всех $i=1, \ldots, N$, то $\gamma_{\Delta}(\kappa)=\gamma(\kappa)$. Можно показать, что $\gamma_{\Delta}$ является емкостью для любого $\Delta \in \mathscr{Z}$. Если $\Lambda$ - направленное множество, $\kappa_{\lambda}, \kappa \subseteq X \times Y$ и $\gamma_{\Delta}(\kappa) \leqslant \liminf _{\lambda \in \Lambda} \gamma_{\Delta}\left(\kappa_{\lambda}\right)\left(\right.$ соответственно $\lim \sup _{\lambda \in \Lambda} \gamma_{\Delta}\left(\kappa_{\lambda}\right) \leqslant$ $\left.\gamma_{\Delta}(\kappa)\right)$ для любого $\Delta \in \mathscr{Z}$, то мы будем писать $\kappa \leqslant \mathrm{c}-\lim \inf \kappa_{\lambda}$ (соответственно $\left.\mathrm{c}-\lim \sup \kappa_{\lambda} \leqslant \kappa\right)$. Будем писать $\kappa=\mathrm{c}-\lim \kappa_{\lambda}$, если $\kappa=\mathrm{c}-\lim \inf \kappa_{\lambda}=\mathrm{c}-\lim \sup \kappa_{\lambda}$.

Пусть $(Z, \tau)$ - топологическое пространство, а $\left\{E_{\lambda}\right\}-$ направленность подмножеств пространства $Z$. Через $\tau$ - $\lim \inf E_{\lambda}$ обозначим множество всех точек $z \in Z$, являющихся $\tau$-пределами направленностей $\left\{z_{\lambda}\right\}$, таких, что $z_{\lambda} \in E_{\lambda}$. Обозначим также через $\tau$-limsup $E_{\lambda}$ множество всех точек $z \in Z$, являющихся точками $\tau$-сгущения таких направленностей. Пишем $E=\tau$ - $\lim E_{\lambda}$, если $E=$ $\tau$ - $\lim \inf E_{\lambda}=\tau$-limsup $E_{\lambda}$. Через w, s* и s обозначим соответственно слабую, *-сильную и сильную операторные топологии на $\mathscr{B}(\mathscr{H}, \mathscr{K})$.

Имеют место следующие результаты, в которых $\kappa$ является $\omega$-замкнутым подмножеством, а $\left\{\kappa_{\lambda}\right\}_{\lambda \in \Lambda}-$ направленностью $\omega$-замкнутых подмножеств в $X \times Y$.

Теорема 1. Положим $S=S_{\kappa}, S_{\lambda}=S_{\kappa_{\lambda}}$. Тогда

(i) Conv $S \subseteq$ w-liminf Conv $S_{\lambda}$ в том и только том случае, когда с-lim $\sup \kappa_{\lambda}$ $\leqslant \kappa$;

(ii) w-limsup Conv $S_{\lambda} \subseteq \operatorname{Conv} S$ в том $u$ только том случае, когда $\kappa \leqslant$ c-liminf $\kappa_{\lambda}$;

(iii) Conv $S=$ w-lim Conv $S_{\lambda}$ в том и только том случае, когда $\kappa=$ с- $\lim \kappa_{\lambda}$. 
Теорема 2. Следующие условия эквивалентны:

(i) $\mathrm{w}-\lim \sup \operatorname{Ball}\left(\mathscr{M}_{\max }\left(\kappa_{\lambda}\right)\right) \subseteq \operatorname{Ball}\left(\mathscr{M}_{\max }(\kappa)\right)$;

(ii) $\mathrm{c}-\lim \sup \kappa_{n} \leqslant \kappa$;

(iii) $S_{\kappa} \subseteq \operatorname{s-liminf} S_{\kappa_{\lambda}}$.

Теорема 3. Рассмотрим следующие утверждения:

(i) $\operatorname{Ball}\left(\mathscr{M}_{\max }(\kappa)\right) \subseteq \mathrm{s}^{*}-\lim \inf \operatorname{Ball}\left(\mathscr{M}_{\max }\left(\kappa_{\lambda}\right)\right)$;

(ii) $\kappa \leqslant c$-liminf $\kappa_{\lambda}$;

(iii) $\mathrm{s}-\lim \sup S_{\kappa_{\lambda}} \subseteq S_{\kappa}$. Тогда (i) $\Longrightarrow$ (ii) $\Longrightarrow$ (iii).

Следствие 4. Предположим, что $\operatorname{Ball}\left(\mathscr{M}_{\max }(\kappa)\right)=\mathrm{s}^{*}-\lim \inf \operatorname{Ball}\left(\mathscr{M}_{\max }\left(\kappa_{\lambda}\right)\right)$ $=\mathrm{w}-\lim \sup \operatorname{Ball}\left(\mathscr{M}_{\max }\left(\kappa_{\lambda}\right)\right)$. Тогда $\kappa=\mathrm{c}-\lim \kappa_{\lambda}$.

Замечания. (i) Импликации (i) $\Longrightarrow$ (iii) в теоремах 2 и 3 обобщают доказанные в [5] и [6] результаты о непрерывности отображения Lat.

(ii) Для $\kappa=\varnothing$ результаты можно сформулировать проще: $\mathrm{w}-\lim \mathscr{M}_{\max }\left(\kappa_{\lambda}\right) \rightarrow$ $\{0\}$ тогда и только тогда, когда $\gamma\left(\kappa_{\lambda}\right) \rightarrow 0$.

(iii) Можно показать, что импликации, обратные к указанным в теореме 3 , не выполняются. Следующий ниже результат представляет собой частичное обращение импликации (ii) $\Longrightarrow$ (i) и устанавливает полунепрерывность снизу отображения $\kappa \rightarrow \mathscr{M}_{\min }(\kappa)$ в более слабом смысле.

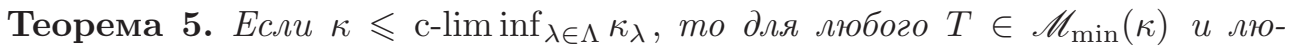
бого счетного набора векторов $\mathscr{E} \subseteq \mathscr{H}$ существует такая направленность $\left\{T_{\lambda}\right\}_{\lambda \in \Lambda}$, что $T_{\lambda} \in \mathscr{M}_{\min }\left(\kappa_{\lambda}\right)$ u $T_{\lambda} x \rightarrow T x$ для любого $x \in \mathscr{E}$.

В частности, если на $\kappa$ выполнен операторный синтез и $\kappa \leqslant \mathrm{c}-\lim \inf _{\lambda \in \Lambda} \kappa_{\lambda}$, то любой элемент из $\mathscr{M}_{\max }(\kappa)$ можно приблизить направленностью $\left\{T_{\lambda}\right\}$, где $T_{\lambda} \in \mathscr{M}_{\text {max }}\left(\kappa_{\lambda}\right)$, на любом счетном множестве векторов.

Благодарность. Авторы признательны профессору В. С. Шульману за плодотворное обсуждение рассматриваемых в статье вопросов.

\section{ЛитерАтУРА}

[1] W. B. Arveson, Ann. of Math. (2), 100 (1974), 433-532. [2] J. A. Erdos, A. Katavolos, V. S. Shulman, J. Funct. Anal., 157:2 (1998), 554-587. [3] R. G. Haydon, V. S. Shulman, Proc. Amer. Math. Soc., 124 (1996), 497-503. [4] А. И. Логинов, В. С. Шульман, Изв. АН СССР, 39:6 (1975), 1260-1273. [5] В. С. Шульман, Функц. анализ и его прил., 23:2 (1989), 86-87. [6] V. S. Shulman, I. G. Todorov, J. Operator Theory, 52:2 (2004), 371-384. [7] V. S. Shulman, L. Turowska, J. Funct. Anal., 209:2 (2004), 293-331.

Department of Pure Mathematics, Queen's University Belfast e-mail: joe.habgood@qub.ac.uk

Поступило в редакцию Department of Pure Mathematics, Queen's University Belfast 7 марта 2007 e-mail: i.todorov@qub.ac.uk 